\title{
Evaluation of six novel antigens as potential biomarkers for the early immunodiagnosis of schistosomiasis
}

Yuanbin Zhang ${ }^{1+}$, Jing Zhao ${ }^{1 \dagger}$, Xinye Wang ${ }^{1}$, Xindong $\mathrm{Xu}^{1 *}$ and Weiqing Pan ${ }^{1,2^{*}}$

\begin{abstract}
Background: Early diagnosis of schistosomiasis, prior to egg laying, would enable earlier treatment and help interrupt the transmission cycle of the parasite and the progress of the disease. Previously we identified six novel antigens with potential as diagnostic markers for human Schistosoma japonicum infections. In this study, we evaluated these antigens as candidate biomarkers for the early diagnosis of schistosomiasis in mice and rabbits.

Methods: The transcriptional profiles of the six antigens (SjSP-13, SjSP-23, SjSP-160, SjSP-164, SjSP-189 and SjSP-216) at different developmental stages were analyzed by quantitative PCR. The recombinant proteins were expressed in E. coli and purified with nickel chelate affinity chromatography. We then developed recombinant protein-based ELISA kits to analyze the kinetics of antigen-specific antibodies during the course of infection in mice and rabbits. The early diagnostic validity of the candidate SjSP-216 was further evaluated in mice and rabbits infected with S. japonicum.

Results: Of the six antigens, SjSP-13, SjSP-160 and SjSP-216 were highly expressed in 21-day old young worms, while SjSP-23, SjSP-164 and SjSP-189 were highly expressed in eggs. In the mouse model, we detected a significant increase in antibodies against SjSP-13 and SjSP-216 at 3 weeks post-infection. However, in the rabbit model, only anti-SjSP-216 antibody showed a significant increase at this time point. We recorded $100 \%$ diagnostic sensitivity and specificity of SSSP-216-based ELISA in both infected mice and rabbits, 3 weeks after infection.
\end{abstract}

Conclusions: This study strongly suggests that SjSP-216, a highly expressed gene in the young worms, could serve as a potential biomarker for the early immunodiagnosis of S. japonicum infections in vertebrate hosts.

Keywords: Schistosoma japonicum, Schistosomiasis, Biomarker, Early diagnosis, Animal model

\section{Background}

Schistosomiasis is a chronic disease caused by parasitic trematodes of the genus Schistosoma that afflicts over 200 million people worldwide and kills $>300,000$ people annually [1-3]. Humans are infected by cercariae, which are released from infected snails when they come in contact with contaminated water [4]. After the cercariae penetrate the skin, the parasites become schistosomula and over 4-6 weeks migrate and mature to adult male or female worms. Adult worms live as pairs in the portal and mesenteric veins (S. japonicum and S. mansoni) or

\footnotetext{
*Correspondence: xuxd@tongji.edu.cn; wqpan0912@aliyun.com

${ }^{\dagger}$ Equal contributors

'Institute for Infectious Diseases and Vaccine Development, Tongji University School of Medicine, Shanghai 200092, China

Full list of author information is available at the end of the article
}

in the veins of the bladder (S. haematobium). They are long-lived and produce hundreds of fertilized eggs per day [5]. Although most eggs are retained within the host tissues, about one-third are excreted into the environment and hatch into free-living miracidia, which can infect susceptible snails in water, thus completing the cycle $[6,7]$. The host's immune response to antigens excreted from the trapped eggs can induce severe morbidity, including hepatic fibrosis, portal hypertension, urinary obstruction and bladder carcinoma $[8,9]$.

One factor contributing to high disease prevalence and severe morbidity is the absence of effective diagnostic methods for detecting schistosome infections, especially in the early phase [10]. The traditional gold standard for the diagnosis of schistosomiasis involves detecting eggs 
in and hatching miracidium from the host's feces or urine $[11,12]$. However, in the majority of schistosome infections, the paired worms start to discharge eggs about 4 weeks after infection [13]. Thus, these diagnostic methods are relatively insensitive and cannot detect early infections. If schistosome infections could be detected prior to egg deposition, the source of infection would be controlled and subsequent treatment with Praziquantel $^{\mathrm{TM}}$ would effectively prevent the development of severe pathologic lesions.

Immunodiagnostic detection of antibodies against schistosome antigens is an attractive option for detecting early infections. However, currently available antibody detection assays are not suitable as they use antigens extracted from schistosome eggs or adult worms [14-16]. Previous studies have revealed that the reactivity of antibodies against crude worm antigens remains low until the infections become patent in the experimental hosts $[17,18]$. Therefore, the ideal immunodiagnostic assay to detect early infections would use antigens from the schistosomula or cercariae [19]. However, it is notoriously difficult to extract antigens from the schistosomula or cercariae.

In a previous study, we used a high-throughput glutathione S-transferase (GST) fusion protein array assay to identify several antigens (SjSP-13, SjSP-23, SjSP-160, SjSP-164, SjSP-189 and SjSP-216) with the potential to be immunodiagnostic markers for human schistosomiasis [20]. In this study, we aim to verify the utility of these six antigens as candidates for the early diagnosis of $S$. japonicum infections in a murine and rabbit model.

\section{Methods}

\section{Parasites and animals}

A field-collected isolate of S. japonicum from Guichi County, Anhui Province, China was used in all the experiments. Parasites were maintained in Oncomelania hupensis snails and in rabbits. Female 12-week old New Zealand White rabbit and female 6-8 week old BALB/c mice were obtained from SLAC Laboratory Animal Co., Ltd. of the Chinese Academy of Sciences of Shanghai. All procedures performed on animals within this study were conducted in accordance with and by approval of the Internal Review Board of Tongji University School of Medicine.

\section{Real-time PCR}

Young worms were recovered by perfusion from BALB/ c mice that had been infected 3 weeks earlier with 200 cercariae. Adult worms were recovered by perfusion from mice 6 weeks post infection. Eggs were purified from livers of infected rabbits. Total RNAs were extracted from cercariae, young worms, adult worms and eggs using Trizol (Invitrogen, USA). First-strand cDNA was performed with the reverse transcriptase Superscript (Takara, Japan) with oligo (dT) primers using $1 \mu \mathrm{g}$ total RNA as template. We then used real-time PCR to quantify gene expression levels. All real-time PCR were run in three replicates. Real-time quantification was performed using an Applied Biosystems 7300 Sequence Detection system using SYBR Premix Ex Taq Kit (Takara). Data were analyzed according to $2^{-\Delta \Delta C t}$ method using GAPDH as the internal control for each sample. The fold-changes of gene transcriptional level in young worm, adult worm and egg were calculated relative to that of cercaria. The house keeping gene SOD was set as a control gene. All primers used for real-time PCR are listed in Table 1.

\section{Cloning, expression and purification of antigens}

The gene fragments of antigens SjSP-13, SjSP-23, SjSP160, SjSP-164, SjSP-189 and SjSP-216 (Genbank accession number: AY222880, AY814664, AY222887, AY814985, AY815838 and AY813624, respectively) were amplified from a mixture of cDNAs of cercariae, young worms, adult worms, and eggs by PCR with KOD polymerase (Toyobo, Japan). Specific primers with restriction enzyme sites are listed in Additional file 1. The PCR products were cloned into E.coli expression vector pGEX-4 T-1 (for SjSP13, SjSP-160, SjSP-164, SjSP-189 and SjSP-216) or pET28a (for SjSP-23). The recombinant plasmids containing target DNA fragments were confirmed by DNA sequencing. Expression of recombinant proteins was induced with Isopropyl-D-1-thiogalactopyranoside (IPTG) at $1 \mathrm{mM}$. Recombinant proteins were purified from the insoluble inclusion body with a hexahistidine tag. The

Table 1 Primers used for Real-Time PCR

\begin{tabular}{ll}
\hline Primer & Primer sequence (5'-3') \\
\hline SjSP-13 Forward & CTGTCGTTACTGTGTGG \\
SjSP-13 Reverse & CCATTCTTCTITTGGAT \\
SjSP-23 Forward & AAGGCGGTATGATTCC \\
SjSP-23 Reverse & CCACGCACTCCTTGTTTCTGA \\
SjSP-160 Forward & GGCGGGCATGGATTTAGTC \\
SjSP-160 Reverse & GCTTGTAATGCCTTGC \\
SjSP-164 Forward & TTCACACACCCTTGGG \\
SjSP-164 Reverse & GTGATGGTGATGGTGATG \\
SjSP-189 Forward & TTAGGGTTCCGATTAGTGC \\
SjSP-189 Reverse & CCGAGATAGGGTTGGTGT \\
SjSP-216 Forward & GGATGCTGGATGGAAAGC \\
SjSP-216 Reverse & GAGGCCATTTCTTCGTG \\
GAPDH Forward & GTGTTCCTACCCCCAATGTGT \\
GAPDH Reverse & GTCATACCAGGAATGAGCTTGA \\
SOD Forward & CTGATGACGGAAAGGGAG \\
SOD Reverse & CTATGACACCACAAGCTACA \\
\hline
\end{tabular}


purified antigens were re-natured in refolding buffer C7 (1.0 mM TCEP, $250 \mathrm{mM} \mathrm{NaCl}, 12.5 \mathrm{mM} \beta$ cyclodextrin, $50 \mathrm{mM}$ Tris- $\mathrm{HCl} \mathrm{pH}$ 8.5) [20]. Protein concentration was determined by the Bradford method [21]. The predicted molecular weight of SjSP-23 and the GST fusion proteins of SjSP-13, SjSP-160, SjSP-164, SjSP-189, SjSP-216 were 13.0kD, 45.6kD, 51.9kD, $40.8 \mathrm{kD}, 41.1 \mathrm{kD}$ and $57.4 \mathrm{kD}$, respectively.

\section{Indirect enzyme-linked immunosorbent assay}

The 96-well microliter plates (Corning, USA) were coated with $100 \mu \mathrm{L}$ per well of 1 to $2 \mu \mathrm{g} / \mathrm{ml}$ antigens diluted in coating buffer $(0.05 \mathrm{M}$ carbonate-bicarbonate, $\mathrm{pH}$ 9.6) for $16 \mathrm{~h}$ at $4{ }^{\circ} \mathrm{C}$. The plates were washed 3 times with washing buffer ( $0.15 \mathrm{M}$ phosphate buffer saline containing $0.05 \%$ of Tween 20, pH 7.4). The free sites were saturated with $200 \mu \mathrm{L}$ per well of blocking buffer (5\% skim milk dissolved in washing buffer) at $37{ }^{\circ} \mathrm{C}$ for $1 \mathrm{~h}$. After washing three times, $100 \mu \mathrm{L}$ of individual mouse sera (diluted 1:100) in blocking buffer were added to the plates and were incubated at $37{ }^{\circ} \mathrm{C}$ for $1 \mathrm{~h}$. The plates were submitted to 5 times of washing and incubated at $37{ }^{\circ} \mathrm{C}$ for $1 \mathrm{~h}$ with goat anti-mouse IgG or goat antirabbit IgG conjugated with peroxidase (Abcam, USA) diluted in blocking buffer at the dilution of 1:20,000. Plates were washed again and $100 \mu \mathrm{L}$ of TMB substrate solution was added to each well. The enzymatic reaction was stopped after $10 \mathrm{~min}$ of incubation at $37{ }^{\circ} \mathrm{C}$ by adding $50 \mu \mathrm{L}$ per well of $2 \mathrm{~N} \mathrm{H}_{2} \mathrm{SO}_{4}$. The results were obtained as absorbance values at $450 \mathrm{~nm}$ by a microplate reader.

\section{Serum collection}

To analyze the dynamics of antigen specific antibodies during infection, three mice and three rabbits were infected with $30 \pm 2$ or $200 \pm 10$ cercariae by the subcutaneous route, respectively. Meanwhile, another three mice and three rabbits were used as non-infected controls. Serum samples were collected before infection and on week 1, 2, 3, 4, 5 and 6 after infection.

A separate experiment was performed to evaluate the early diagnostic validity of SjSP-216. Ten mice and six rabbits were infected the same as before; ten mice and six rabbits were used as a negative control of infection. Sera were collected at 3 weeks after infection.

\section{Statistic analysis}

Data of real-time PCR were expressed as the mean \pm standard deviation. The absorbance values were expressed as the mean \pm standard error. All data were analyzed by the Student's $t$-test, with a $p$ value $<0.05$ considered significant. We used Prism 4.0 software for all statistical analyses.

\section{Results}

\section{Production of recombinant proteins}

The gene sequences of the six proteins were verified as correct by DNA sequencing. The six recombinant proteins were successfully produced in E.coli (Additional file 2). The purified antigens were re-natured in refolding buffer C7 that was identified from the iFOLD Protein Refolding System 1 in our previous studies [20].

\section{Analysis of transcriptional profiles of the six antigens at different developmental stages}

The gene transcriptional profiles of the six antigens varied within the different developmental stages (Fig. 1). SjSP-13 (Fig. 1a), SjSP-160 (Fig. 1c) and SjSP-216 (Fig. 1f) were highly expressed in young worms and adult worms but poorly expressed in eggs. Conversely, the expression levels of SjSP-164 (Fig. 1d), SjSP-189 (Fig. 1e), and particularly SjSP-23 (Fig. 1b) were higher at the egg stage than the other stages. None of the six antigens were highly expressed in cercariae. As expected, the transcriptional level of the control gene, SOD, was similar at all developmental stages (Additional file 3).

\section{Kinetics of specific antibodies in the sera of mice infected with S. japonicum}

Sera from three infected mice were collected before infection ( 0 week) and at week 1 to 6 weeks post-infection. Three uninfected mice were used as negative controls. We found that the levels of specific antibodies against all six antigens increased during the course of infection in mouse but that the seroconversion times differed for each antigen (Fig. 2). We detected a significant increase in antibodies against SjSP-13 and SjSP-216 3 weeks post-infection (Fig. 2a and f). This was 1 week earlier than SjSP-160 (Fig. 2c), SjSP-164 (Fig. 2d), and SjSP-189 (Fig. 2e) and 3 weeks earlier than SjSP-23 (Fig. 2b). These results indicated that SjSP-13 and SjSP-216 are candidates for early serological diagnosis in mice.

\section{Kinetics of specific antibodies in the sera of rabbits infected with S. japonicum}

We observed a significant increase in antibodies against SjSP-13, SjSP-23, SjSP-160 and SjSP-216 in the three infected rabbits 6 weeks post-infection (Fig. 3). There was no increase in antibodies against SjSP-164 and SjSP-189 during the course of infection (Fig. 3). As in the mouse host, antibodies against SjSP-216 increased significantly just 3 weeks after infection (Fig. 3f). Significant increases in antibodies against SjSP-13 did not occur until 5 weeks post-infection (Fig. 3a). Interestingly, antibodies against SjSP-23 could only be detected 6 weeks post-infection in both the mouse and the rabbit model. Hence, these results indicated only SjSP-216 could serve as a candidate for early diagnosis of schistosomiasis in rabbits. 


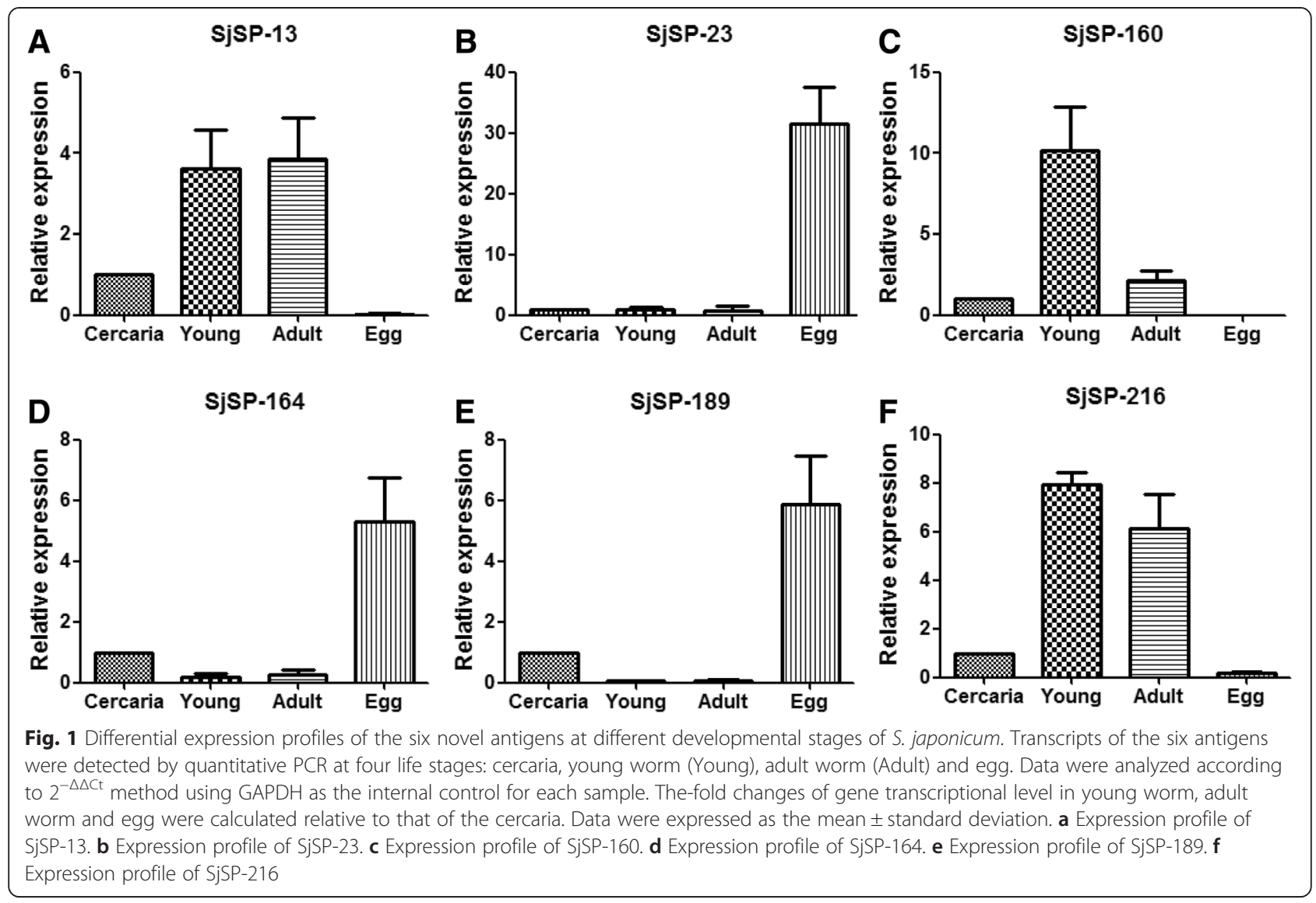

Sensitivity and specificity of SjSP-216 as a candidate for early serological diagnosis of $S$. japonicum infections

The results from the above experiments indicated that the SjSP-216 protein had the potential to be a protein marker for early schistosome infection. To further investigate its diagnostic validity of early infection, we carried out additional experiments in the murine and rabbit models of human schistosmiasis with a large sample size (ten mice and six rabbits for both infection group and non-infection group). As shown in Fig. 4, in the mouse experiment, all the serum samples collected from the infected mice at 3 weeks after infection yielded a seropositive reaction (100\% seroconversion) whereas the samples from the uninfected mice showed no seroreaction. Similar to the results in mice, all the serum samples from the infected rabbits at three weeks after infection generated seropositive reactions (100\% seroconversion) and no SjSP-216 specific antibody was detectable in the uninfected rabbits.

\section{Discussion}

Schistosomiasis remains a serious public health problem in developing countries. Morbidity and mortality from this disease are associated with the chronic infection stage, which occurs after egg deposition. Thus, an ideal diagnostic test for schistosomiasis should be capable of detecting parasites as early as possible after the onset of infection. An early diagnostic test would enable more rapid treatment and would interrupt the transmission cycle of the parasite and the progress of the disease. However, the current diagnostic standards for schistosomiasis all depend on the detection of eggs. In addition, as disease is asymptomatic in its early stages, clinical examinations can't confirm infection. Recently, molecular techniques to detect schistosome infections have been developed to facilitate early diagnosis, but these are expensive and suffer from sampling limitations [22, 23]. Serologic assays to detect antibodies against schistosome antigens, however, have proven useful in the clinical diagnosis of schistosomiasis [24-26].

In definitive hosts, the immune systems must confront four life-cycle stages of the parasite: penetrating cercariae, schistosomula (young worms), adult worms and the eggs that are produced by adult worm pairs [8]. As the host is exposed (internally) to cercariae and schistosomula earlier than they are exposed to eggs, antigens that are highly expressed or solely expressed in one of these two stages are likely be potential candidates for early diagnosis. For 

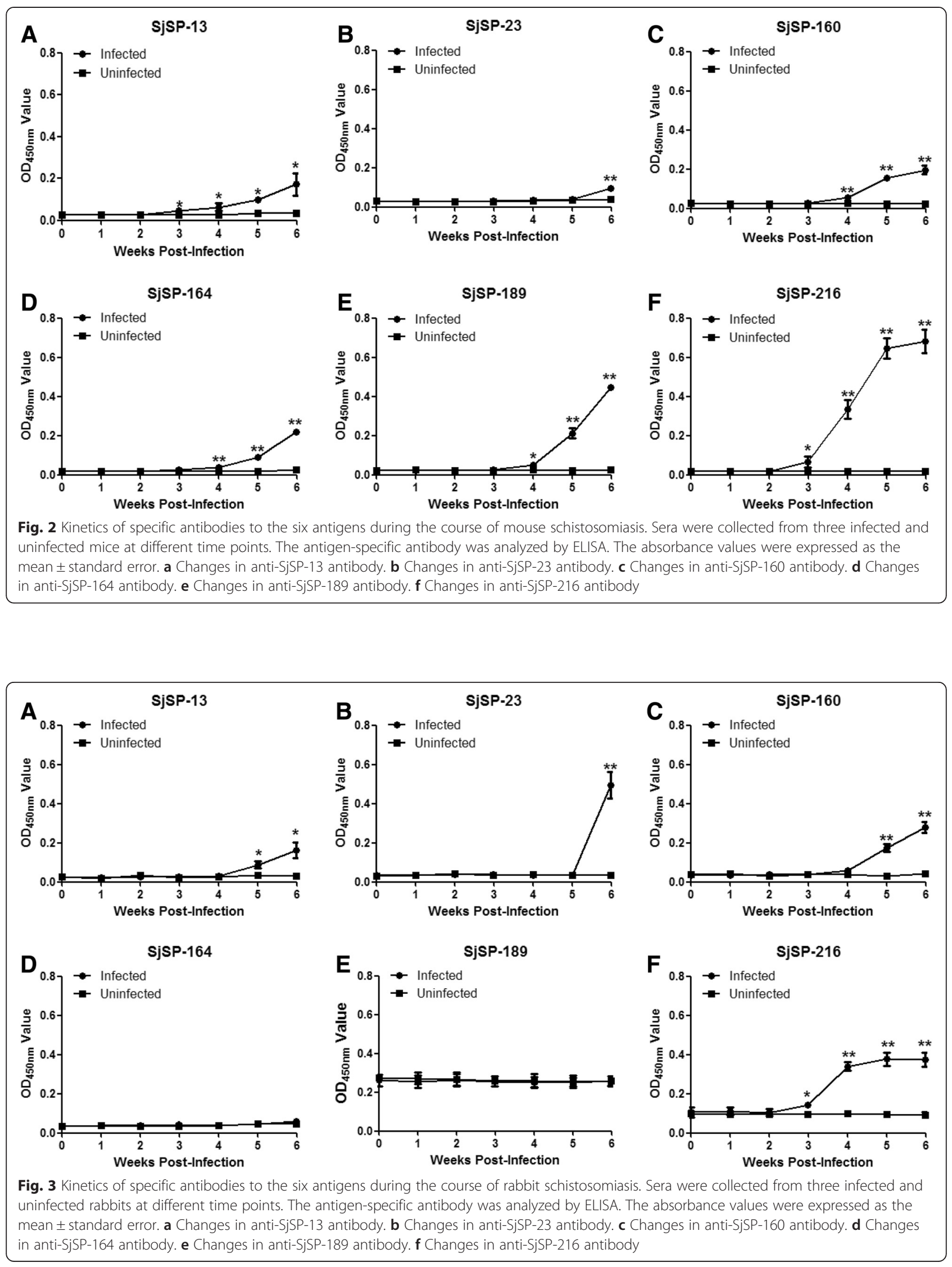


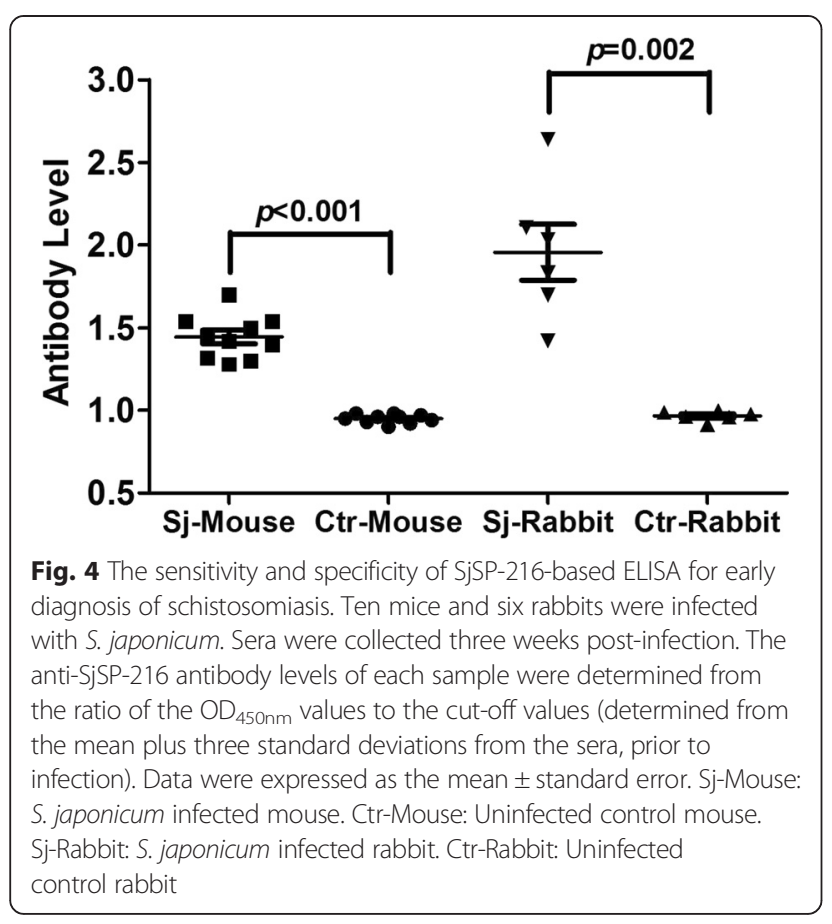

example, previous studies of the schistosomula (mechanically transformed cercariae) tegument antigen revealed this antigen had diagnostic value for early infections [19]. In this study, we found that antigens SjSP-13, SjSP-160 and SjSP-216 were highly expressed in young worms, while antigens, SjSP-23, SjSP-164 and SjSP-189 were highly expressed in eggs. These distinct expression profiles correlate with the timing of host immune responses to specific stages. For example, seroconversion of young worm antigen SjSP-216 occurred 3 weeks post-infection, 3 weeks earlier than to the egg antigen SjSP-23.

The immunogenicity of antigens varied between the two host species. Previously, we found that SjSP-164 and SjSP-189 were recognized by the antibodies of human patients [20]. In this study, we report that SjSP-164 and SjSP-189 stimulated humoral immune responses in infected mice but not in rabbits. We found that SjSP-13, which has been verified as a diagnostic marker for human schistosomiasis [20], is also recognized by IgG antibodies in both infected mice and rabbits. Nevertheless, the titers of antibodies in mice and rabbits were much lower than those reported for humans, indicating that the antigenicity of SjSP-13 was weaker in these species than in humans. Importantly, the antigenicity of SjSP-216 in mice and rabbits appeared very similar to that reported for this antigen in humans.

For these reasons, we selected the antigen, SjSP-216, as a candidate for early diagnosis of schistosomiasis. Our results confirmed that SjSP-216 was able to diagnose early schistosome infections in both mice and rabbits with 100
$\%$ sensitivity and specificity. Moreover, we observed that anti-SjSP-216 antibody levels increased throughout the period of the parasite exposure. Thus, SjSP-216 would also be useful for diagnosing chronic infections. Although early diagnosis may not be necessary in areas with high endemicity where most patients harbor chronic infections [27], it is vital for detecting new infections, and for ensuring effective disease surveillance in areas where the schistosomiasis has be controlled. In areas where schistosomiasis has been controlled, large-scale disease surveillance at the start of the transmission season could identify (and remove) sources of infection and prevent the recurrence of schistosomiasis. Moreover, early diagnosis will lead to earlier treatment, potentially preventing the development of severe pathologic lesions. This would be particularly relevant for susceptible individuals, such as travellers [28]. Although Praziquantel ${ }^{\mathrm{TM}}$ is more effective on adult worms than young worms, high-dose $(500 \mathrm{mg} / \mathrm{kg})$ Praziquantel $^{\mathrm{TM}}$ therapy or prolonging the course of treatment can damage the tegument or even kill young worms [29, 30].

We propose that SjSP-216 be further developed as an early serological diagnostic tool for human schistosomiasis, as this protein has strong antigenicity in different hosts, including humans. It is difficult to validate the early diagnostic efficacy of SjSP-216 in humans directly as current techniques can't detect pre-patent human infections. In addition, it is not easy to regularly monitor antibody changes during the course of infection in human. However, it is possible to compare the positive rates of SjSP-216 and other antigens including SjSP-13 at the start of transmission season (i.e. in May) and at the end of epidemic season (i.e. in September). If the sensitivity of SjSP-216 was higher during the epidemic season, we can prove the early diagnostic efficacy of SjSP-216 indirectly.

\section{Conclusions}

This study strongly suggests that SjSP-216, a highly expressed gene in the young worm stage, could serve as a potential biomarker for the early immunodiagnosis of S. japonicum infections in vertebrate hosts.

\section{Additional files}

Additional file 1: Primers used for gene cloning. (PDF $29 \mathrm{~kb}$ )

Additional file 2: SDS-PAGE analysis of purified recombinant proteins. Proteins were expressed in E. coli and purified using the Ni-NTA agarose affinity system under denaturing conditions. The purified antigens were re-natured in refolding buffer C7 before SDS-PAGE analysis. (PDF $117 \mathrm{~kb}$ )

Additional file 3: Expression profile of superoxide dismutase (SOD) gene at different developmental stages of S. japonicum. Real-time PCR was performed to detect the transcripts of SOD at life stages of cercaria, young worm (Young), adult worm (Adult) and egg. Data were analyzed according to $2-\mathrm{Ct}$ method using GAPDH as the internal control for each sample. The-fold changes of gene transcriptional level in 
young worm, adult worm and egg were calculated as compared with cercaria. (PDF $73 \mathrm{~kb}$ )

\section{Competing interests}

The authors declare that they have no competing interests.

\section{Authors' contributions}

YZ and IZ carried out the experimental work, interpreted and analyzed the data. XW provided significant support to animal experiments. XX conceived the project, interpreted and analyzed the data, and wrote the manuscript. WP conceived the project and critically revised the manuscript. All authors read and approved the final manuscript.

\section{Acknowledgements}

This study was supported by grants from the National Natural Science Foundation of China (81430051) and the Shanghai Municipal Natural Science Foundation (15ZR1443500).

\section{Author details}

${ }^{1}$ Institute for Infectious Diseases and Vaccine Development, Tongji University School of Medicine, Shanghai 200092, China. ${ }^{2}$ Department of Tropical Infectious Diseases, Second Military Medical University, Shanghai 200433, China.

\section{Received: 14 July 2015 Accepted: 17 August 2015}

\section{Published online: 04 September 2015}

\section{References}

1. Gryseels B, Polman K, Clerinx J, Kestens L. Human schistosomiasis. Lancet. 2006;368(9541):1106-18.

2. Gray DJ, McManus DP, Li Y, Williams GM, Bergquist R, Ross AG. Schistosomiasis elimination: lessons from the past guide the future. Lancet Infect Dis. 2010;10(10):733-6.

3. King CH, Dickman K, Tisch DJ. Reassessment of the cost of chronic helmintic infection: a meta-analysis of disability-related outcomes in endemic schistosomiasis. Lancet. 2005;365(9470):1561-9.

4. Grimes JE, Croll D, Harrison WE, Utzinger J, Freeman MC, Templeton MR. The roles of water, sanitation and hygiene in reducing schistosomiasis: a review. Parasit Vectors. 2015:8:156.

5. Chabasse D, Bertrand G, Leroux JP, Gauthey N, Hocquet P. [Developmental bilharziasis caused by Schistosoma mansoni discovered 37 years after infestation]. Bull Soc Pathol Exot Filiales. 1985;78(5):643-7.

6. Cheever AW, Mosimann JE, Deb S, Cheever EA, Duvall RH. Natural history of Schistosoma mansoni infection in mice: egg production, egg passage in the feces, and contribution of host and parasite death to changes in worm numbers. Am J Trop Med Hyg. 1994;50(3):269-80.

7. Cheever AW, Macedonia JG, Mosimann JE, Cheever EA. Kinetics of egg production and egg excretion by Schistosoma mansoni and S. japonicum in mice infected with a single pair of worms. Am J Trop Med Hyg. 1994;50(3):281-95.

8. Colley DG, Secor WE. Immunology of human schistosomiasis. Parasite Immunol. 2014;36(8):347-57.

9. Steinmann P, Keiser J, Bos R, Tanner M, Utzinger J. Schistosomiasis and water resources development: systematic review, meta-analysis, and estimates of people at risk. Lancet Infect Dis. 2006;6(7):411-25.

10. Bergquist $R$, Johansen MV, Utzinger J. Diagnostic dilemmas in helminthology: what tools to use and when? Trends Parasitol. 2009;25(4):151-6.

11. Yu JM, de Vlas SJ, Jiang QW, Gryseels B. Comparison of the Kato-Katz technique, hatching test and indirect hemagglutination assay (IHA) for the diagnosis of Schistosoma japonicum infection in China. Parasitol Int. 2007:56(1):45-9.

12. Katz N, Chaves A, Pellegrino J. A simple device for quantitative stool thicksmear technique in Schistosomiasis mansoni. Rev Inst Med Trop Sao Paulo. 1972;14(6):397-400.

13. Barbosa MA, Pellegrino J, Coelho PM, Sampaio IB. Quantitative aspects of the migration and evolutive asynchronism of Schistosoma mansoni in mice. Rev Inst Med Trop Sao Paulo. 1978;20(3):121-32.

14. Zhou YB, Yang MX, Tao P, Jiang QL, Zhao GM, Wei JG, et al. A longitudinal study of comparison of the Kato-Katz technique and indirect hemagglutination assay (IHA) for the detection of schistosomiasis japonica in China, 2001-2006. Acta Trop. 2008;107(3):251-4.

15. Zhu YC. Immunodiagnosis and its role in schistosomiasis control in China: a review. Acta Trop. 2005;96(2-3):130-6.

16. Doenhoff MJ, Chiodini PL, Hamilton JV. Specific and sensitive diagnosis of schistosome infection: can it be done with antibodies? Trends Parasitol. 2004;20(1):35-9.

17. Ambroise-Thomas P, Andrews P. Development of fluorescent antibodies directed against larval stages, eggs, and adults of Schistosoma mansoni in mice harbouring unisexual or bisexual infections. Tropenmed Parasitol. 1976;27(4):483-8.

18. Dunne DW, Bain J, Lillywhite J, Doenhoff MJ. The stage-, strain- and species-specificity of a Schistosoma mansoni egg antigen fraction (CEF6) with serodiagnostic potential. Trans R Soc Trop Med Hyg. 1984;78(4):460-70.

19. Grenfell R, Martins W, Silva-Moraes V, Araujo N, Oliveira E, Fonseca C, et al. The schistosomula tegument antigen as a potential candidate for the early serological diagnosis of schistosomiasis mansoni. Rev Inst Med Trop Sao Paulo. 2013;55(2):75-8.

20. Xu X, Zhang Y, Lin D, Zhang J, Xu J, Liu YM, et al. Serodiagnosis of Schistosoma japonicum infection: genome-wide identification of a protein marker, and assessment of its diagnostic validity in a field study in China. Lancet Infect Dis. 2014;14(6):489-97.

21. Stoscheck CM. Quantitation of protein. Methods Enzymol. 1990;182:50-68.

22. Xu J, Rong R, Zhang HQ, Shi CJ, Zhu XQ, Xia CM. Sensitive and rapid detection of Schistosoma japonicum DNA by loop-mediated isothermal amplification (LAMP). Int J Parasitol. 2010;40(3):327-31.

23. Suzuki T, Osada Y, Kumagai T, Hamada A, Okuzawa E, Kanazawa T. Early detection of Schistosoma mansoni infection by touchdown PCR in a mouse model. Parasitol Int. 2006;55(3):213-8.

24. Lin DD, Xu JM, Zhang YY, Liu YM, Hu F, Xu XL, et al. Evaluation of IgG-ELISA for the diagnosis of Schistosoma japonicum in a high prevalence, low intensity endemic area of China. Acta Trop. 2008;107(2):128-33.

25. Tsang VC, Hillyer GV, Noh J, Vivas-Gonzalez BE, Ahn LH, Pilcher JB, et al. Geographic clustering and seroprevalence of schistosomiasis in Puerto Rico (1995). Am J Trop Med Hyg. 1997;56(1):107-12

26. Cai YC, XU JF, Steinmann P, Chen SH, Chu YH, Tian LG, et al. Field comparison of circulating antibody assays versus circulating antigen assays for the detection of schistosomiasis japonica in endemic areas of China. Parasit Vectors. 2014;7:138.

27. Zhou XN, Guo JG, Wu XH, Jiang QW, Zheng J, Dang H, et al. Epidemiology of schistosomiasis in the People's Republic of China, 2004. Emerg Infect Dis. 2007;13(10):1470-6.

28. Coltart CE, Chew A, Storrar N, Armstrong M, Suff N, Morris L, et al. Schistosomiasis presenting in travellers: a 15 year observational study at the Hospital for Tropical Diseases, London. Trans R Soc Trop Med Hyg. 2015;109(3):214-20.

29. Shaw MK. Schistosoma mansoni: stage-dependent damage after in vivo treatment with praziquantel. Parasitology. 1990;100(Pt 1):65-72.

30. Xiao SH, Shen BG. Scanning electron microscope observation on tegumental damage of 21-d-old Schistosoma japonicum induced by praziquantel. Zhongguo Yao Li Xue Bao. 1995;16(3):273-5.

\section{Submit your next manuscript to BioMed Central and take full advantage of:}

- Convenient online submission

- Thorough peer review

- No space constraints or color figure charges

- Immediate publication on acceptance

- Inclusion in PubMed, CAS, Scopus and Google Scholar

- Research which is freely available for redistribution 